\title{
Temporal properties in masking biological motion
}

\author{
ERIC HIRIS, DEVON HUMPHREY, and ALEXANDRA STOUT \\ St. Mary's College of Maryland, St. Mary's City, Maryland
}

\begin{abstract}
The perception of biological motion using point light animation techniques was investigated in several experiments. Animations simulating walking were presented with additional masking dots. The temporal properties of the walking motion or the temporal relationship between the walking and masking motions were systematically manipulated. Results showed that (1) perception of biological motion was sensitive to even small temporal perturbation within the walker, (2) the effectiveness of a mask depended upon the temporal phase difference between the mask and point light walker, (3) relatively small temporal differences between the mask and point light walker decreased the effectiveness of the mask, and (4) these effects were not due simply to observers detecting the phase offsets in the display. Temporal properties of the motion are important in perceiving the human form in action, just as in other types of figure-ground segregation. This information may be processed by both motion and form pathways for processing biological motion.
\end{abstract}

The motions of 10 to 12 points of light placed on the major joints of the body are sufficient to give the strong impression of the human form and the nature of the activity being performed (Johansson, 1973). Such biological motion displays offer a rich source of information about the action and the actor portrayed. Potentially, they can carry information about the gender of the actor (Barclay, Cutting, \& Kozlowski, 1978; Kozlowski \& Cutting, 1977, 1978; Mather \& Murdoch, 1994; Pollick, Lestou, Ryu, \& Cho, 2002; Runeson \& Frykholm, 1983; Troje, 2002), the identity of the actor (Beardsworth \& Buckner, 1981; Cutting \& Kozlowski, 1977; Hill \& Pollick, 2000; Stevenage, Nixon, \& Vince, 1999), the effort of the action (Runeson \& Frykholm, 1981), the emotions of the actor (Brownlow, Dixon, Egbert, \& Radcliffe, 1997; Dittrich, Troscianko, Lea, \& Morgan, 1996; Pollick et al., 2002; Pollick, Paterson, Bruderlin, \& Sanford, 2001; Walk \& Homan, 1984), the expectations and intentions of the actor (Runeson \& Frykholm, 1983), or the size of the actor (Jokisch \& Troje, 2003); they can even carry information for the perception of sign language (Poizner, Bellugi, \& Lutes-Driscol, 1981). Inverting biological motion displays, however, even though it preserves the relationships between the moving dots, is known to disrupt the perception of biological motion (Grossman \& Blake, 2001; Mitkin \& Pavlova, 1990; Pavlova \& Sokolov, 2000; Shipley, 2003; Sumi, 1984; Troje, 2003). The effect of inversion on biological motion detection may be due to the unfamiliar form that the inverted point lights now create

A portion of this work was presented at the 3rd Annual Meeting of the Vision Science Society, Sarasota, FL in 2003. We thank L. Kimberly Epting, Martin Giese, and two anonymous reviewers for comments on an earlier draft of this manuscript. Correspondence should be addressed to E. Hiris, Department of Psychology, St. Mary's College of Maryland, 18952 E. Fisher Rd., St. Mary's City, MD 20686 (e-mail: ejhiris@smcm.edu).
(Bertenthal \& Pinto, 1994) or to unfamiliar dynamics in relation to gravity (Shipley, 2003).

One common way to investigate biological motion is to mask the displays by simultaneously presenting other moving points of light in the display area. Cutting, Moore, and Morrison (1988) showed that a scrambled walker mask disrupted perception of an embedded point light walker more effectively than did translating masks, circular masks, or random motion masks. Furthermore, a scrambled walker mask with the same step-size as that of the walker maximizes disruption, and with a sufficient number of mask elements, performance does not improve with presentations longer than $800 \mathrm{msec}$.

In detecting masked biological motion, the central task is to segregate the figure (biological motion) from the ground (the masking dots). Figure-ground segregation can be supported by many cues (e.g., orientation, color, depth, motion). However, in masked biological motion, the walker and mask dots have the same orientation, color, depth, and so on. As Cutting et al. (1988) showed, in general the more similar the motion of the mask dots to the motion of the walker dots, the more effective the mask. This is not surprising, given that as the similarity of the mask and walker dot motions increase, there are fewer cues to support figure-ground segregation. In fact, Bertenthal and Pinto (1994) argue that using a scrambled walker mask requires global processing of biological motion by rendering local processing of the dot motions uninformative. Furthermore, detecting biological motion within a scrambled walker mask demands greater attentional load than does detecting biological motion within a random motion mask (Thornton, Rensink, \& Shiffrar, 2002).

Temporal coherence is known to play a role in figureground segregation. Fahle (1993) showed that time differences as small as $5 \mathrm{msec}$ were sufficient to discriminate the shape of a rectangle defined solely by the phase differences between figure and ground elements. Segre- 
gation based on temporal phase differences can even combine with other segregation cues (Leonards, Singer, \& Fahle, 1996). Indeed, visual form can be created solely from synchronized changes in nonoverlapping structures (Lee \& Blake, 1999). Form, however, also influences coherence judgments. Tadin, Lappin, Blake, and Grossman (2002) showed that coherence judgments were better when the local elements carrying the coherence signal were organized as a global moving form, such as upright biological motion, than when the local elements created inverted biological motion.

Previous research has investigated the effects of spatial and temporal perturbations of biological motion on its detectability (i.e., the ability to segregate figure from ground). Spatial perturbation of biological motion results from placing the dots off the joints of the moving figure, for example, in the middle of the forearm rather than on the elbow. This manipulation has only a small effect on the detectability of the walker (Bertenthal \& Pinto, 1994; Cutting, 1981; Dittrich, 1993), suggesting that spatially perturbed walkers provide an adequate representation of biological motion. Temporal perturbation of biological motion results when individual dots within the walker are randomly shifted in phase within the stepcycle. ${ }^{1}$ These displays are more difficult to detect than are normal or spatially perturbed walkers within masks, suggesting that the temporal properties of the motion are more critical for perceiving biological motion (Bertenthal \& Pinto, 1994).

Given that detecting biological motion within a mask is essentially a figure-ground segregation task and that temporal coherence is an effective cue for figure-ground segregation, we sought to determine how temporal coherence affected the perception of masked biological motion. The first experiment addressed how a range of temporal phase offsets within biological motion affected the detectability of biological motion. The remaining experiments addressed how temporal phase offsets between the mask and the walker (i.e., temporal coherence of the type investigated in the figure-ground segregation literature) affected the detectability of biological motion.

\section{EXPERIMENT 1}

Experiments involving temporal perturbations of the walker motions have demonstrated clearly the importance of phase information in perceiving biological motion. Phase information can be used as the basis of a discrimination task in which observers must discriminate between normal in-phase walkers and phase-scrambled walkers (Ahlstrom, Blake, \& Ahlstrom, 1997; Blake, 1993; Blake, Turner, Smoski, Pozdol, \& Stone, 2003; Grossman \& Blake, 1999). Alternatively, phase information can be used as a manipulation to affect detectability within a mask (Bertenthal \& Pinto, 1994; Cutting et al., 1988). However, in both uses of phase manipulations, researchers have used either completely random or very large temporal perturbations to demonstrate the effect of phase manipulation. Experiment 1 sought to determine how much temporal perturbation within the walker is required to disrupt the perception of biological motion.

\section{Method}

Observers. Ten naive observers with normal or corrected-tonormal visual acuity participated in the experiment. All observers were introductory psychology students who received course credit for their participation.

Stimuli. The stimuli were point light walkers embedded within scrambled walker masks. Point light walkers were created synthetically using a modified version of Cutting's (1978) technique. The custom-written software generated 360 frames of motion for each

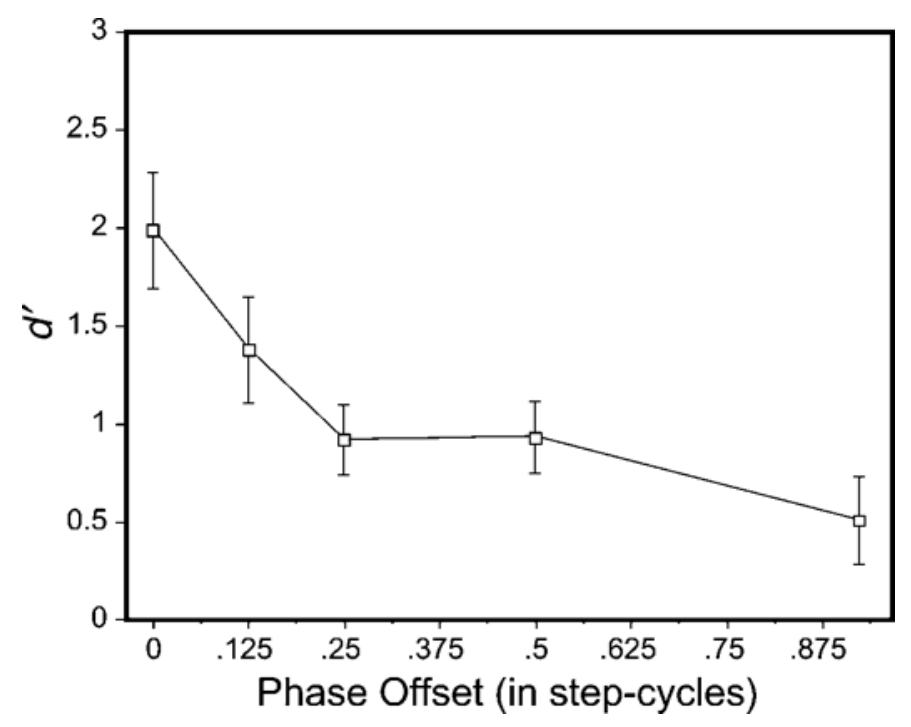

Figure 1. Results from Experiment 1. The abscissa gives the phase offset range in step-cycles within the point light walker. The ordinate shows the average $d^{\prime}$ for the observers. Error bars represent \pm 1 standard error of the mean. 
step cycle of walking. Although only 40 frames of motion were used to present a step-cycle, dots from any of the 360 frames generated could be used, depending on the stimulus condition. Eleven dots were visible in the side view of the left-facing walker, and no occlusion cues were present in the display. The starting position of the dots within the step-cycle was randomized from trial to trial. One complete step-cycle was presented on each trial.

Temporal coherence of the walker dots was manipulated by changing the phase offset range (in terms of step-cycle) within the walker. For example, if the phase offset was 0 , all walker dots were on the same part of the step-cycle; that is, the walker exhibited complete temporal coherence. If the phase offset was .5 step-cycles, each walker dot was offset by an amount between 0 and .5 stepcycles. Phase offset amounts were distributed among the walker dots so that they were evenly distributed throughout the range. For example, when the phase offset was .5 step-cycles, the 11 walker dots were offset by $0, .05, .1, .15, .2, .25, .3, .35, .4, .45$, and .5 stepcycles. Assignment of phase offsets to particular dots was random from trial to trial.

A scrambled walker mask was created by randomly plotting 88 dots within the display area, with each mask dot moving in the same manner as one of the dots in the walker. The motion of the mask dots was determined after the phase offset of the walker dots, so the motion of the mask dots and walker dots always matched exactly. To avoid possible local density difference for target-present and target-absent trials, additional mask dots were not plotted randomly when the walker was not present. Instead, the additional mask dots appeared at the same location where the walker dots would have appeared but had different motions assigned to them. Consequently, a static view of the first frame of the motion sequence was not sufficient to determine whether the walker was present or absent. This method of replacing the walker dots was always used in this and the other experiments reported here.

Custom-written software running on a Power Macintosh G4 computer with a Mitsubishi 22 -in. monitor $(1600 \times 1200$ pixels at $85 \mathrm{~Hz}$ ) was used to present the displays. A head and chinrest was used to enforce a $57-\mathrm{cm}$ viewing distance. At this viewing distance, the dots subtended approximately $0.2^{\circ}$ of visual angle, whereas the walker subtended $8.1^{\circ}$ in height and $4.1^{\circ}$ in width at the point of greatest extent. The mask dots were displayed within an $18.8^{\circ}$ high $\times 15.1^{\circ}$ wide area. The walker was centered within the mask area, but its exact location varied by $3.75^{\circ}$ vertically and horizontally from trial to trial. One step-cycle was completed in 40 frames and was presented at a rate of 42.5 frames per second.

Procedure. The observers completed five blocks of 50 trials corresponding to phase offsets of $0, .125, .25, .5$, and .92 step-cycles. Note that given the cyclical nature of walking, a phase offset of .92 results in the 11 walker dots' being evenly distributed throughout the step-cycle. This is the most temporally disturbed walker possible when the dots are uniformly distributed throughout the phase. The observers' task was to indicate whether or not the walker was present within the mask. The walker was present in $50 \%$ of the trials. The order of the blocks was partially counterbalanced across observers.

Before each block of trials, a five-step practice procedure familiarized each observer with the stimuli on which he or she was to be tested. First, the observers were shown a point light walker with no mask in five separate presentations of one step-cycle and were asked to describe what they saw. If an observer did not describe a person walking, he or she was told that the display was meant to represent a person walking, and step 1 was repeated. Second, the observers received instructions for starting a trial (by pressing the space key) and responding to a trial (by pressing the " $\mathrm{f}$ " key if a person walking was not present and the " $\mathrm{j}$ " key if a person walking was present). Small signs reminding the observers which key corresponded with which response were attached to the black mask that surrounded the computer monitor. After receiving these instructions, the observers viewed and responded to 10 trials where the walker was present on $50 \%$ of the trials. Third, the observers performed 10 trials with masks where the walker was present on $50 \%$ of the trials. In this set of trials, the walker dots were always white and the mask dots always gray. The observers were told that although the difference in dot colors makes it trivially easy to detect the presence of a walker, these trials give the opportunity to become familiar with the appearance of walker dots presented within mask dots. Fourth, the observers performed 10 trials where both walker and mask dots were white (i.e., identical to the trials in the main experiment). Fifth, the observers again were shown the unmasked version of the stimulus and were reminded that this was the "target" stimulus that they were to identify as present or absent within the mask during the main experiment. The observers could repeat any of the practice procedures if desired.

\section{Results and Discussion}

Detection sensitivity for each observer in each condition was calculated as $d^{\prime}$ by the methods outlined in Macmillan and Creelman (1991). A repeated measures analysis of variance (ANOVA) was conducted with stepcycle offset amount as the independent variable and $d^{\prime}$ as the dependent measure. The effect of phase offset was significant [using the Greenhouse-Geisser correction for sphericity; $F(3.09,27.79)=8.00, \eta^{2}=.471, p<$ $.001]$. As the phase offset within the walker increased, the presence of the walker became more difficult to detect. As Figure 1 shows, most of this increased difficulty comes from changing the phase offset range from 0 to .25 step-cycles. Note that here each of the 11 walker dots is within about .02 step-cycles of at least one other walker dot in the display. Previous researchers have shown that large temporal perturbations disrupt biological motion perception. The present data demonstrate that the visual system is sensitive to even small temporal perturbations of walking motions.

\section{EXPERIMENT 2A}

In Experiment 1, observers had greater difficulty detecting biological motion as the amount of temporal perturbation within the walker increased. However, temporally perturbing the walker also changes the form of the walker. Several observers in Experiment 1 noted that its limbs looked rubbery or that the walker did not look "normal." This change in the form of the target within the mask makes it difficult to conclude that the difference in detection sensitivity is due to temporal perturbations per se (an aspect of motion) and not form. Experiment $2 \mathrm{~A}$ tested the temporal sensitivity of the visual system in a way that did not disturb the form of the walker. Instead of manipulating the phase offsets within the walker, the phase difference between the mask and walker dots was manipulated. Sufficiently large phase differences between the mask and walker dots should provide robust figure-ground segregation cues, allowing the observer to more easily detect the masked biological motion. Inverted, as well as upright, biological motion displays were used in the experiment. Inverted biological motion displays are less recognizable (e.g., Sumi, 1984), and presumably the phase differences would be 


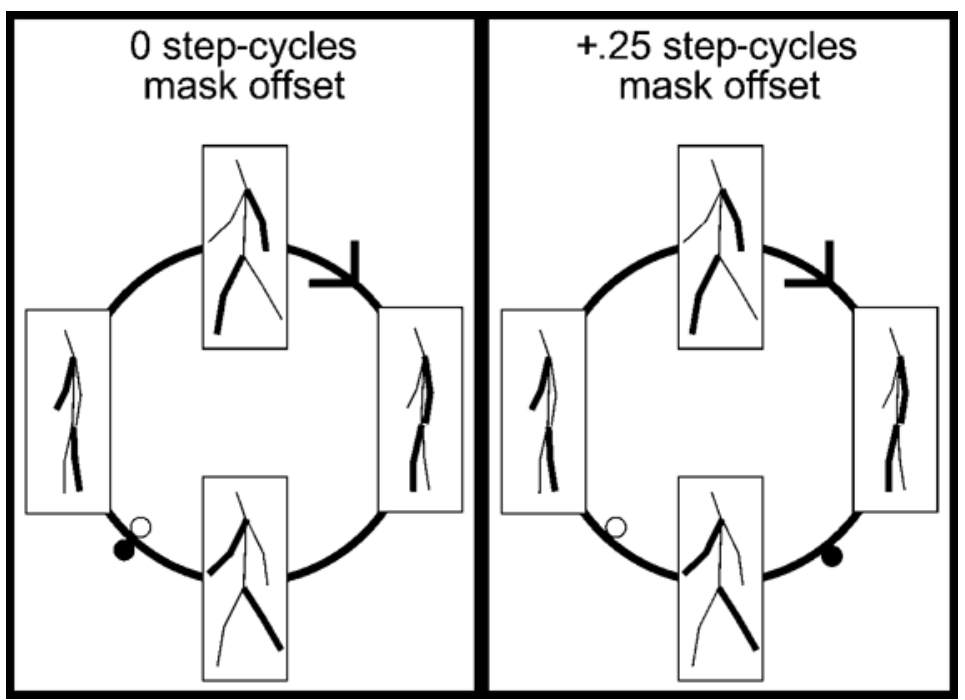

Figure 2. Schematic of mask phase offsets. The large circle represents one step-cycle of walking. Stick figures show the position of the body at four points in the step-cycle. Limbs on the left side of the body are bold for clarity. The temporal relationship between the mask and walker dots in the step-cycle are given by the relative position of the open circle (mask dots) and filled circle (walker dots). The left panel shows a mask phase offset of 0 step-cycles. The right panel illustrates a mask phase offset of +.25 step-cycles.

less helpful in segregating this poorly organized "form" from the background.

\footnotetext{
Method

Observers. Eighteen naive observers with normal or correctedto-normal visual acuity participated in the experiment. All observers were introductory psychology students who received course credit for their participation.

Stimuli. The stimuli were the same as those described in Experiment 1 , except for three changes. First, the walker was centered within the mask area, but the exact location of the walker within the mask was varied by $5.0^{\circ}$ vertically and horizontally from trial to trial (rather than $3.75^{\circ}$ ). The walker location was varied more in this experiment to avoid ceiling effects, given that we provided a temporal coherence cue for figure-ground segregation that should make the task easier rather than more difficult (cf. disrupting the coherence of the walker in Experiment 1). Variability in location of $5.0^{\circ}$ was the maximum usable in the displays without the motion of the walker dots being truncated. Second, both upright and inverted walkers were used. Third, rather than manipulating the phase offset within the walker, we manipulated the phase of the mask dots relative to the walker dots. Each mask dot still moved in the same manner as did one of the walker dots, but did so either slightly before or after the corresponding walker dot made the same motion. Figure 2 illustrates two different mask phase offsets: a mask phase offset of 0 step-cycles ( 0 frame offset in the cycle of 360 frames) where the corresponding mask and walker dots move in the same way at the same time and a mask phase offset of +.25 step-cycles $(90$ frame offset in the cycle of 360 frames) where the corresponding mask dots move in the same way as the walker dots but before the walker dots have done so.

Procedure. The observers completed nine blocks of 50 trials over 2 days (four blocks the 1 st day, five blocks the 2 nd day). The nine blocks corresponded to mask phase offsets of $-180,-135,-90$, $-45,0,45,90,135$, and 180 frames ( -.5 to +.5 step-cycles). Each
}

observer was assigned to either upright or inverted walkers and completed blocks of trials for all mask phase offsets. The observers' task was to indicate whether or not the walker was present within the mask. The walker was present on $50 \%$ of the trials. The order of the blocks was partially counterbalanced across participants. Before each block of trials, each observer was familiarized with the stimuli for this experiment using the five-step practice procedure described in Experiment 1 .

\section{Results and Discussion}

Detection sensitivity was calculated as in Experiment 1 for each observer in each condition. A mixed factors ANOVA was conducted with mask phase offset as the within-subjects variable, walker orientation as the between-subjects variable, and $d^{\prime}$ as the dependent measure. There were three main findings from the experiment. First, inverted walkers were more difficult to detect than upright walkers for all mask phase offsets $\left[F(1,16)=13.99, \eta^{2}=.18, p<.005\right]$. Second, there was an effect of mask phase offset [using the GreenhouseGeisser correction for sphericity; $F(5.10,81.65)=$ $\left.26.06, \eta^{2}=.45, p<.001\right]$. Specifically, mask phase offsets of $0,-.5$, and .5 step-cycles resulted in similarly low performance in comparison with other mask phase offset conditions, which resulted in better discriminability. This division into two groups $(0,-.5$, and .5 step-cycles vs. the remaining conditions) was verified by a Tukey's HSD test. Third, the effect of the mask phase offset was less pronounced on inverted walkers than on upright walkers [significant interaction using the GreenhouseGeisser correction for sphericity; $F(5.10,81.65)=3.04$, $\left.\eta^{2}=.05, p<.05\right]$. 
Note that a mask phase offset of 0 is the standard scrambled walker mask stimulus. Given that the mask and walker dots are moving the same way at the same time, it perhaps is not surprising that this mask phase offset results in poor performance. Performance was equally poor in the -.5 and .5 mask phase offset conditions. This can be understood by considering that the mask dots here are exactly half of a step-cycle out of synchrony with the walker dots, resulting in mask dots that are nominally associated with the left side of the walker masking the motion of the dots associated with the right side of the walker (compare the stick figure at the bottom and top of the step-cycle in Figure 2). Because of this, data from the appropriate negative and positive mask offsets were combined for presentation in the upper panel of Figure 3. Note that a mask phase offset of .25 maximizes the difference between mask and walker dots. This is exactly where the local peaks of the function are in the upper panel of Figure 3.

The decrease in the detectability of inverted walkers replicates previous findings regarding the inversion of biological motion (e.g., see Bertenthal \& Pinto, 1994; Shipley, 2003; Sumi, 1984; Troje, 2003). Yet even inverted walkers are easier to detect with some mask phase offsets, although improvement is not as great as for upright walkers. This finding is consistent with the notion that some form information, albeit degraded, about inverted walkers is available to the visual system (e.g., see Tadin et al., 2002).

The upper panel of Figure 3 also shows a large jump in performance between the most difficult conditions (mask phase offsets of 0 and .5) and the neighboring condition tested (.125 or .375). Although it is clear that mask phase offsets can influence the detectability of a point light walker, it is not clear how much temporal perturbation between the walker and the mask is necessary to affect detectability.

\section{EXPERIMENT 2B}

In this experiment, the mask phase offset parameter was sampled more finely to determine where mask phase offsets begin to affect biological motion detection. Because of the equality of positive and negative mask offsets, only positive mask offsets were used.

\section{Method}

Observers. Two sets of observers were used. Both sets were naive to the purpose of this study and had normal or corrected-to-normal visual acuity. One set consisted of 3 observers who had previous experience in biological motion experiments and were available for extensive data-collection sessions. These 3 observers were paid for their participation. The second set consisted of 8 observers with no previous experience with biological motion experiments. These observers were introductory psychology students who received course credit for their participation and were available for only two 30-min data-collection sessions.

Stimuli. The stimuli were the same as those used in Experiment $2 \mathrm{~A}$. For the experienced observers, the following mask phase offset values were used: $0,1,2,4,8,16,32,45,60,75,90,135$, and
180 frames ( 0 to .5 step-cycles). For the inexperienced observers, the following mask phase offset values were used: $0,1,2,4,8,16$, 32 , and 45 frames ( 0 to .125 step-cycles).

Procedure. Each experienced observer completed two runs of 50 trials for both upright and inverted versions of the display and was presented with all 13 mask phase offset values, for a total of 52 blocks ( 2 runs $\times 2$ orientations $\times 13$ mask step-cycle offset values). Blocks were completed in groups of four or five per session over several weeks. The blocks were run in quasirandom order for all observers, each of whom completed an initial run on each condition before any condition was run a second time. Each inexperienced observer completed eight blocks of 50 trials over two days (four

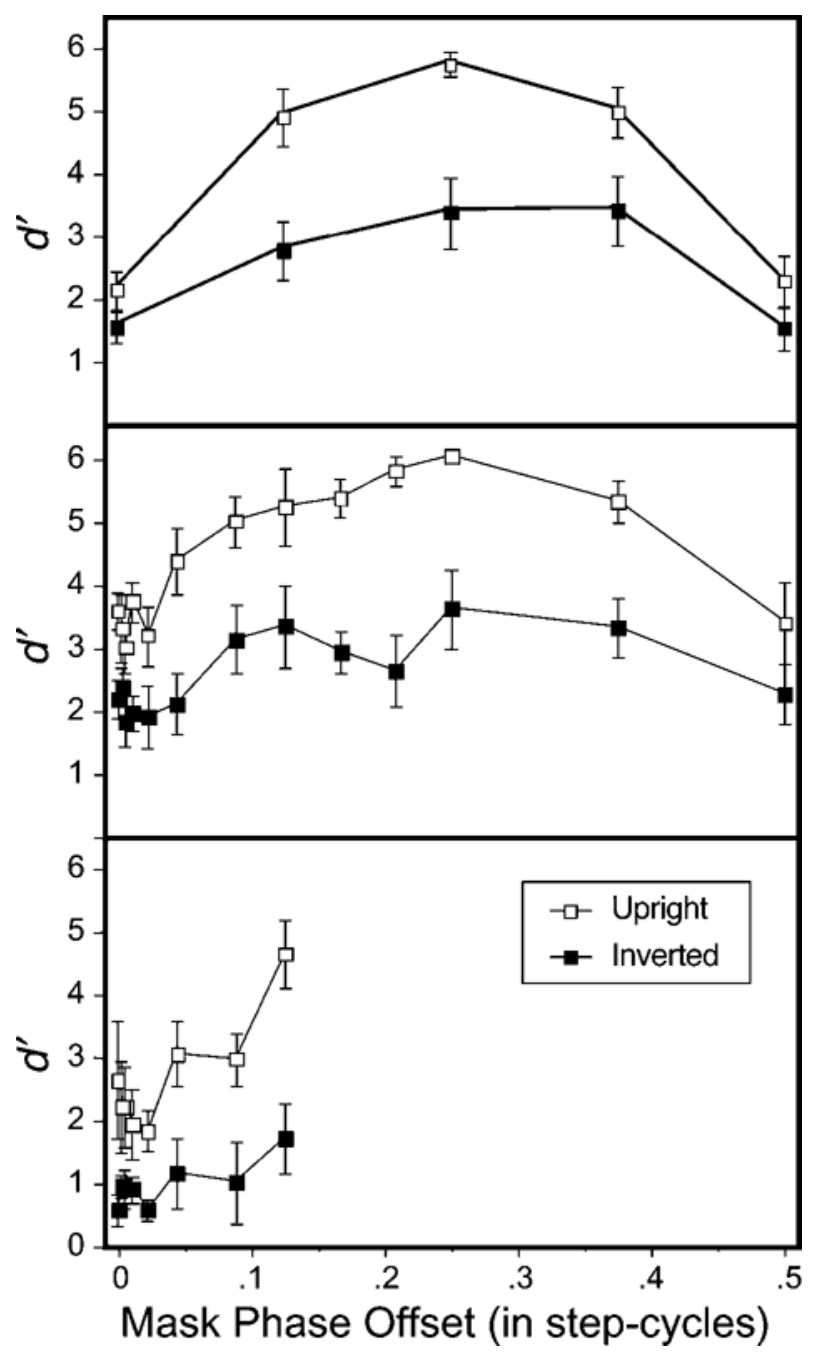

Figure 3. Results from Experiments $2 \mathrm{~A}$ and 2B. The abscissa gives the mask phase offset in step-cycles. Each ordinate shows the average $d^{\prime}$ for the observers of that experiment. Error bars represent \pm 1 standard error of the mean. Upper panel: The combined results from positive and negative mask phase offsets for Experiment 2A. For this panel, the abscissa shows the results for the 0 mask phase offset condition as well as the averaged data from the following conditions: \pm .5 step-cycles, +.375 and -0.125 step-cycles, \pm .25 step-cycles, +.125 and -.375 step-cycles. See text for details. Middle panel: The results from the experienced observers in Experiment 2B. Lower panel: The results from the naive observers in Experiment 2B. 
blocks per day). All observers were instructed to indicate the presence or absence of the walker within the mask. The walker was present on $50 \%$ of the trials. Before each block of trials, each observer was familiarized with the stimuli for his or her next block of trials using the five-step practice procedure described in Experiment 1 .

\section{Results and Discussion}

Detection sensitivity was calculated as in Experiment 1 for each observer in each condition. For the data from experienced observers, a nonadditive repeated measures ANOVA was conducted with the mask phase offset, walker orientation, and run number as independent variables and $d^{\prime}$ as the dependent measure. For the data from inexperienced observers, a mixed factors ANOVA was conducted with mask phase offset as the within-subjects factor, walker orientation as the betweensubjects factor, and $d^{\prime}$ as the dependent measure.

The middle panel of Figure 3 plots the data for upright and inverted walkers for all the mask phase offsets for experienced observers. The lower panel of Figure 3 plots the same information for the inexperienced observers. These panels illustrate two of three main findings from the experiment. First, inverted walkers again proved more difficult to detect than upright walkers across all mask offsets for both experienced observers $\left[F(1,2)=187.02, \eta^{2}=.26, p<.01\right]$ and inexperienced observers $\left[F(1,6)=24.01, \eta^{2}=.38, p<.005\right]$. Second, but not shown in the middle panel of Figure 3 , there was an effect of run number, with the second run having $d^{\prime}$, on average, 0.9 higher than the first run, indicating substantial improvement on the task with experience $\left[F(1,2)=36.34, \eta^{2}=.08, p<.05\right]$. Third, there was an effect of mask phase offset for both experienced observers [using the Greenhouse-Geisser correction for sphericity; $\left.F(1.97,3.95)=11.62, \eta^{2}=.35, p<.05\right]$ and inexperienced observers [using the Greenhouse-Geisser correction for sphericity; $F(3.22,19.30)=3.29, \eta^{2}=$ $.19, p<.05]$. None of the interactions between the other variables were significant for experienced or inexperienced observers, including the interaction between mask phase offset and walker orientation. This interaction was significant in Experiment 2A but likely was not significant in this experiment because many small mask phase offset values were tested and the difference in performance between upright and inverted walkers is relatively constant for these small mask phase offset values.

The effect of mask phase offset was investigated further using post hoc tests. For experienced observers, Dunnett's post hoc test (using the 0 mask phase offset condition as the control comparison) showed that performance improved with mask phase offsets of 32 frames (about .09 step-cycles) and greater, suggesting that the visual system is highly sensitive to the temporal perturbation between mask and walker dots. Note, however, that .09 step-cycles corresponds to a time delay of about $95 \mathrm{msec}$, significantly longer than temporal offsets necessary for figure-ground segregation of around $10 \mathrm{msec}$ (Fahle, 1993; Leonards et al., 1996). Of course, this difference may be explained by the fact that we measured when segregation improved, not the minimum temporal offset necessary to create segregation. For inexperienced observers, Dunnett's test showed that performance improved when the mask phase offset was 45 frames (.125 step-cycles). Note that the mask phase offset value that results in improved performance was nearly the same for experienced and inexperienced observers. This suggests that the effect of temporal perturbations between the walker and mask dots on biological motion detection does not depend on extensive previous exposure to biological motion displays. Nonetheless, comparison of the middle and lower panels of Figure 3 shows that experienced observers in general perform better than naive ones. On average, experienced observers had a $d^{\prime}$ value that was larger by 1 . This, perhaps, makes it more remarkable that performance, in comparison with baseline, appears to change at approximately the same mask phase offset value.

\section{EXPERIMENT 3}

Experiments $2 \mathrm{~A}$ and $2 \mathrm{~B}$ showed that mask phase offsets enabled observers to detect the walker more easily for both upright and inverted displays. Leonards et al. (1996) showed that temporal phase differences can be used in combination with other segregation cues. If we eliminate all form cues from the display-that is, even the residual cues available in inverted displays - we can determine how critical form is for the use of temporal coherence as a segregation cue in biological motion displays. In Experiment 3, we tested this possibility by presenting observers with targets defined only by phase offsets. If observers detect the presence of a phase offset without an upright or inverted walker as well as they do with one present, performance in Experiments $2 \mathrm{~A}$ and 2B may have been driven solely by detecting phase offsets. On the other hand, if observers cannot detect the presence of a phase offset without an upright or inverted walker, performance in Experiments $2 \mathrm{~A}$ and $2 \mathrm{~B}$ were based at least in part on segregation cues from both phase offset and form information.

\section{Method}

Observers. Ten naive observers with normal or corrected-tonormal visual acuity participated in the experiment. All observers were introductory psychology students who received course credit for their participation.

Stimuli. The stimuli were created on the basis of the motion of a walker, although no walker was ever presented in this experiment. Target-absent trials were the same as those in Experiment 2A-that is, mask dots moving with no phase offset with any other dots in the display. However, on target-present trials, no walker was present; instead, a scrambled version of the walker was presented with a phase offset between the scrambled walker dots and the mask dots. Therefore, these scrambled walker dots moved in the same way as the rest of the dots on the screen except slightly afterward (see Figure 2). Under these conditions, the only way to distinguish between target absence and target presence was to detect the presence of a phase offset. Given the pattern of results in the previous experiments and that the greatest functional phase offset is .25 (see Ex- 
periment $2 \mathrm{~A}$ ), the following phase offset values were used: 8,16 , 32,45 , and 90 frames (.02-.25 step-cycles). Although the target in the display, a scrambled walker, did not look like a recognizable figure, both upright and inverted versions were used. Here, upright and inverted refer to the motions in the source walker from which the target, if present, and the mask derived their motions. Half of the participants performed the experiment with upright motion and half with inverted motion.

Procedure. All five mask phase offset values were tested in a single session with the order of the blocks partially counterbalanced across observers. Each observer completed 50 trials for each mask phase offset value. The target was present on $50 \%$ of the trials. Given that the target is defined by its relationship to the other dots in the display, the five-step familiarization procedure was modified into a four-step procedure. In the first step, the target was shown in white dots among gray mask dots. In the second step, the observers responded to 10 trials (50\% target present) of the stimulus with the same white target dots and gray mask dots as in Step 1. In the third step, all dots were white and 10 more practice trials were performed. In the fourth and final step, the observers again saw the target with white dots and the mask with gray dots. Before the experiment began, the observers were asked whether they wanted to repeat any of the practice conditions.

\section{Results and Discussion}

Detection sensitivity was calculated as in Experiment 1 for each observer in each condition. A mixed factors ANOVA was conducted with mask phase offset as the within-subjects factor, target orientation as the between-subjects factor, and $d^{\prime}$ as the dependent measure. Figure 4 plots the data for upright and inverted targets for all mask phase offsets. Given that the target dots did not represent a recognizable figure, it is not surprising that there was no significant difference between upright and inverted targets. There also was no significant interaction between orientation and mask phase offset. Note that unlike in the previous experiments, most data fall near a $d^{\prime}$ of 0 , indicating that observers had great difficulty perceiving a target solely defined by a phase offset. However, there was nearly a significant effect of mask phase offset [using the Greenhouse-Geisser correction for sphericity; $\left.F(2.18,17.46)=2.98, \eta^{2}=.24, p<.08\right]$. Apparently, observers can detect, at best, only the largest phase offsets in the absence of a coherent target stimulus. These data suggest that observers in Experiments $2 \mathrm{~A}$ and $2 \mathrm{~B}$ were not basing their judgments solely on the detection of mask phase offset, but instead were also processing the information about the form of the biological motion. The consistent significant difference between upright and inverted walkers found in Experiments $2 \mathrm{~A}$ and $2 \mathrm{~B}$ also supports this interpretation. There would be no reason to expect a difference between upright and inverted walkers if the observers were basing their judgments solely on the phase offset in the display.

\section{GENERAL DISCUSSION}

The results of Experiment 1 showed that point light walkers with phase-shifted dots are more difficult to detect than point light walkers without phase-shifted dots. This finding is congruent with previous work (e.g., Bertenthal \& Pinto, 1994). In addition, Experiment 1 revealed that much of the effect of phase shifting within a walker occurs with phase-shift ranges as small as .25 step-cycles. Experiments 2A and 2B showed that point light walkers are easier to detect within a mask if there is a phase offset between the walker and mask dots. Disrupting the form of the walker by inversion (Experiments $2 \mathrm{~A}$ and $2 \mathrm{~B}$ ) or temporal perturbation (Experiment 1)

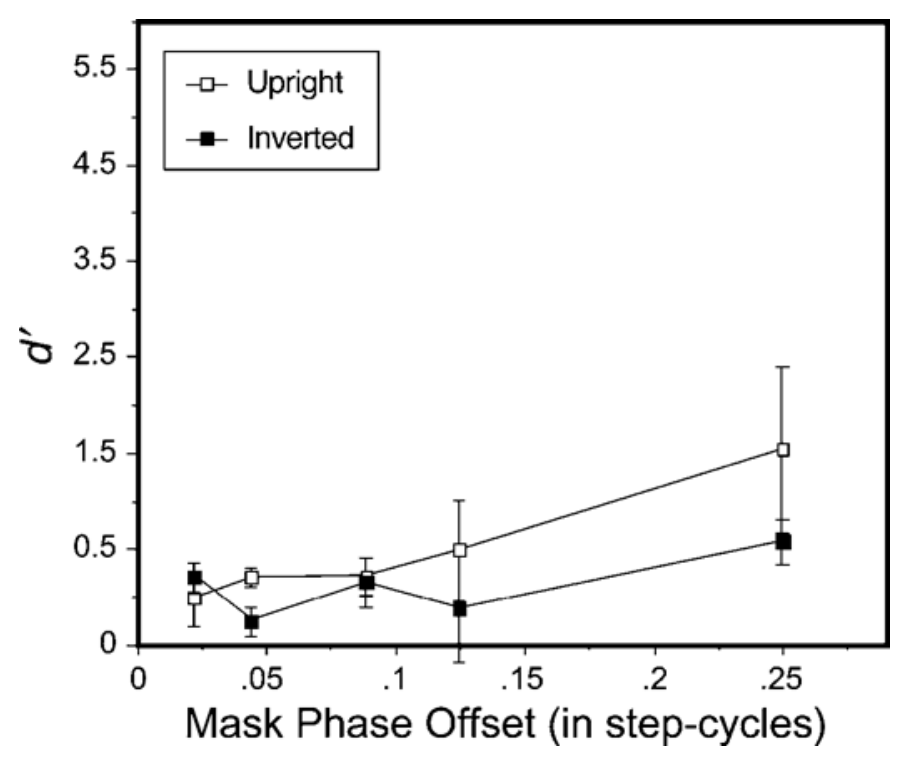

Figure 4. Results from Experiment 3. The abscissa gives the phase offset of the mask dots in comparison with the target dots in step-cycles. The ordinate shows the average $d^{\prime}$ for the observers. Error bars represent \pm 1 standard error of the mean. 
makes it more difficult to detect. Finally, Experiment 3 showed that performance in Experiments 2A and 2B could not be explained by detection of the phase offset itself. These experiments show that the visual system is very sensitive to temporal perturbations within biological motion and between the walker and masking dots. Temporal coherence and form information appear to be used in combination to segregate the figure (biological motion) from the ground (masking dots). This supports previous work suggesting that multiple cues can be used in combination to segregate figure and ground (Leonards et al., 1996).

Giese and Poggio (2003) have proposed a physiologically plausible model of biological motion perception that processes form and motion information in two pathways. Given the dynamic nature of biological motion stimuli, including a motion information pathway in the model is obvious. Certainly, it is well established that motion information influences the detectability and recognizability of the displays (Barclay et al., 1978; Johansson, 1976; Mather \& Murdoch, 1994; Shiffrar, Lichtey, \& Chatterjee, 1997). Several lines of research support the inclusion of form information in a model of biological motion perception. Recently, Beintema and Lappe (2002) presented biological motion displays in which the walker was defined by eight points of light randomly positioned along the limb from frame to frame, strongly degrading local motion cues. Even with degraded local motion cues, observers could perceive the biological motion stimulus. However, recent models of biological motion suggest that even when motion is locally degraded, the motion pathway is more active than the form pathway (Casile \& Giese, 2003). Whether this is a consequence of the particular model used or a reflection of how humans process biological motion remains to be determined.

It is clearer, however, that form information or motion selective structures outside the lesioned areas are being used in individuals with damage to low-level motion mechanisms who are still able to perceive biological motion (McLeod, Dittrich, Driver, Perret, \& Zihl, 1996; Vaina, Lemay, Bienfang, Choi, \& Nakayama, 1990). In addition, functional magnetic resonance imaging research has shown that the perception of human motion involves many cortical areas such as the superior temporal sulcus, and occipital and fusiform face areas (Bonda, Petrides, Ostry, \& Evans, 1996; Grezes et al., 2001; Grossman \& Blake, 2002; Grossman et al., 2000; Servos, Osu, Santi, \& Kawato, 2002). The lateral occipital complex and extrastriate body area are involved in the perception of human form, but it is not clear that these areas respond to biological motion. Downing, Yuhong, Shuman, and Kanwisher (2001) found a significant difference in the responses to biological motion displays and scrambled control displays in the extrastriate body area. However, others have not been able to replicate this finding (Grossman \& Blake, 2002).

How can the data from the present experiments be reconciled with a model of biological motion perception in which both form and motion information are important?
In Experiment 1, disturbing the temporal relations between the dots that defined the walker made the walker more difficult to detect. Initially, one might attribute this to degradation of motion information in the display. However, several observers noted that the walking motion appeared "rubbery" or "not normal." In fact, many of the displays resulted in anatomically impossible motions of the lower limbs relative to the upper limbs. For example, the lower arm sometimes appeared to move backward at the elbow as if to touch the back of the shoulder, rather than the front. Therefore, these "temporally perturbed" displays degraded both form and motion information. Given a model that relies on both form and motion information, perhaps it is not surprising that performance decreased quickly when both motion and form information were disrupted in Experiment 1 (see Figure 1). Experiments $2 \mathrm{~A}$ and $2 \mathrm{~B}$ showed that efficacy of the mask depended upon the mask phase offset. It is clear that manipulating only the mask phase offset does not perturb the form of the walker. However, Experiment 3 showed that phase offset alone cannot be detected reliably, indicating that motion information alone cannot account for the effect of the mask phase offset in Experiments $2 \mathrm{~A}$ and 2B. Apparently then, including additional moving dots along with a point light walker masks not only biological motion, but its form as well. Mask phase offsets, like those used in Experiments 2A and 2B, may function as a motion-based cue to the embedded point light walker form.

Overall, the present experiments demonstrate that biological motion perception is sensitive to the temporal properties of the display, although this sensitivity to motion information may rely on processing by both motion and form pathways. It remains unclear exactly how the visual system uses form and motion information to process biological motion (cf. Bertenthal \& Pinto, 1994; Shipley, 2003), but a framework for conducting such investigations has recently emerged (Giese \& Poggio, 2003). One goal of future research should be to understand the relative contributions of form and motion information in perceiving biological motion, as well as the extent to which these contributions may depend upon viewing conditions or task demands.

\section{REFERENCES}

Ahlstrom, V., Blake, R., \& Ahlstrom, U. (1997). Perception of biological motion. Perception, 26, 1539-1548.

Barclay, C. D., Cutting, J. E., \& Kozlowski, L. T. (1978). Temporal and spatial factors in gait perception that influence gender recognition. Perception \& Psychophysics, 23, 145-152.

BEARDSWORTH, T., \& BUCKNER, T. (1981). The ability to recognize oneself from a video recording of one's movements without seeing one's body. Bulletin of the Psychonomic Society, 18, 19-22.

Beintema, J. A., \& LapPe, M. (2002). Perception of biological motion without local image motion. Proceedings of the National Academy of Sciences, 99, 5661-5663.

Bertenthal, B. I., \& Pinto, J. (1994). Global processing of biological motions. Psychological Science, 5, 221-225.

BlaKe, R. (1993). Cats perceive biological motion. Psychological Science, 4, 54-57.

Blake, R., Turner, L. M., Smoski, M. J., Pozdol, S. L., \& Stone, 
W. L. (2003). Visual recognition of biological motion is impaired in children with autism. Psychological Science, 14, 151-157.

Bonda, E., Petrides, M., Ostry, D., \& Evans, A. (1996). Specific involvement of human parietal systems and the amygdala in the perception of biological motion. Journal of Neuroscience, 16, 37373744.

Brownlow, S., Dixon, A. R., Egbert, C. A., \& Radcliffe, R. D. (1997). Perception of movement and dancer characteristics from point-light displays of dance. Psychological Record, 47, 411-421.

Casile, A., \& Giese, M. (2003). Roles of motion and form in biological motion recognition. Lecture Notes in Computer Science, 2714, $854-862$.

Cutting, J. E. (1978). A program to generate synthetic walkers as dynamic point-light displays. Behavior Research Methods \& Instrumentation, 10, 91-94.

CutTing, J. E. (1981). Coding theory applied to gait perception. Journal of Experimental Psychology: Human Perception \& Performance, 7, 71-87.

CutTing, J. E., \& Kozlowski, L. T. (1977). Recognizing friends by their walk: Gait perception without familiarity cues. Bulletin of the Psychonomic Society, 9, 353-356.

Cutting, J. E., Moore, C., \& Morrison, R. (1988). Masking the motions of human gait. Perception \& Psychophysics, 44, 339-347.

DitTRICH, W. H. (1993). Action categories and the perception of biological motion. Perception, 22, 15-22.

Dittrich, W. H., Troscianko, T., Lea, S. E. G., \& Morgan, D. (1996). Perception of emotion from dynamic point-light displays represented in dance. Perception, 25, 727-738.

Downing, P. E., Yuhong, J., Shuman, M., \& Kanwisher, N. (2001). A cortical area selective for visual processing of the human body. Science, 293, 2470-2473.

FAHLE, M. (1993). Figure-ground discrimination from temporal information. Proceedings of the Royal Society of London: Series B, 254, 199-203.

Giese, M. A., \& Poggio, T. (2003). Neural mechanisms for the recognition of biological movements. Nature Reviews Neuroscience, $\mathbf{4}$, 179-192.

Grezes, J., Fonlupt, P., Bertenthal, B., Delon-Martin, C., SegeBARTH, C., \& DeCETY, J. (2001). Does perception of biological motion rely on specific brain regions? NeuroImage, 13, 775-785.

Grossman, E. D., \& Blake, R. (1999). Perception of coherent motion, biological motion and form-from-motion under dim-light conditions. Vision Research, 39, 3721-3727.

Grossman, E. D., \& BlaKe, R. (2001). Brain activity evoked by inverted and imagined biological motion. Vision Research, 41, 1475-1482.

Grossman, E. D., \& BlaKe, R. (2002). Brain areas active during visual perception of biological motion. Neuron, 35, 1167-1175.

Grossman, E. [D.], Donnelly, M., Price, R., Pickens, D., MorGan, V., Neighbor, G., \& Blake, R. (2000). Brain areas involved in perception of biological motion. Journal of Cognitive Neuroscience, 12, 711-720.

HiLl, H., \& Pollick, F. E. (2000). Exaggerating temporal differences enhances recognition of individuals from point light displays. Psychological Science, 11, 223-228.

Johansson, G. (1973). Visual perception of biological motion and a model for its analysis. Perception \& Psychophysics, 14, 201-211.

JoHansson, G. (1976). Spatio-temporal differentiation and integration on visual motion perception. Psychological Research, 38, 379-393.

Jokisch, D., \& Troje, N. F. (2003). Biological motion as a cue for the perception of size. Journal of Vision, 3, 252-264.

Kozlowski, L. T., \& CUtTING, J. E. (1977). Recognizing the sex of a walker from a dynamic point-light display. Perception \& Psychophysics, 21, 575-580.

KozlowsKi, L. T., \& CUtTing, J. E. (1978). Recognizing the gender of walkers from point-lights mounted on ankles: Some second thoughts. Perception \& Psychophysics, 23, 459.

LEE, S., \& BlaKe, R. (1999). Visual form created solely from temporal structure. Science, 284, 1165-1168.

Leonards, U., Singer, W., \& Fahle, M. (1996). The influence of tem- poral phase differences on texture segmentation. Vision Research, 36, 2689-2697.

Macmillan, N. A., \& Creelman, C. D. (1991). Detection theory: A user's guide. New York: Cambridge University Press.

Mather, G., \& Murdoch, L. (1994). Gender discrimination in biological motion displays based on dynamic cues. Proceedings of the Royal Society of London: Series B, 258, 273-279.

McLeod, P., Dittrich, W., Driver, J., Perret, D., \& Zihl, J. (1996). Preserved and impaired detection of structure from motion by a "motionblind" patient. Visual Cognition, 3, 363-391.

Mitkin, A. A., \& Pavlova, M. A. (1990). Changing a natural orientation: Recognition of biological motion pattern by children and adults. Psychologische Beiträge, 32, 28-35.

Pavlova, M., \& SoKolov, A. (2000). Orientation specificity in biological motion perception. Perception \& Psychophysics, 62, 889-899.

Poizner, H., Bellugi, U., \& Lutes-Driscol, V. (1981). Perception of American sign language in dynamic point-light displays. Journal of Experimental Psychology: Human Perception \& Performance, 7, 430-440.

Pollick, F. E., Lestou, V., Ryu, J., \& Cho, S. (2002). Estimating the efficiency of recognizing gender and affect from biological motion. Vision Research, 42, 2345-2355.

Pollick, F. E., Paterson, H. M., Bruderlin, A., \& Sanford, A. J. (2001). Perceiving affect from arm movement. Cognition, 82, B51-B61.

Runeson, S., \& Frykholm, G. (1981). Visual perception of lifted weights. Journal of Experimental Psychology: Human Perception \& Performance, 7, 733-740.

Runeson, S., \& Frykholm, G. (1983). Kinematic specifications of dynamics as an informational basis for person and action perception: Expectation, gender-recognition, and deceptive intention. Journal of Experimental Psychology: General, 112, 585-615.

Servos, P., Osu, R., Santi, A., \& Kawato, M. (2002). The neural substrates of biological motion perception: An fMRI study. Cerebral Cortex, 12, 772-782.

Shiffrar, M., Lichtey, L., \& ChatterJee, S. H. (1997). The perception of biological motion across apertures. Perception \& Psychophysics, 59, 51-59.

SHIPLEY, T. F. (2003). The effect of object and event orientation on perception of biological motion. Psychological Science, 14, 377-380.

Stevenage, S. V., Nixon, M. S., \& Vince, K. (1999). Visual analysis of gait as a cue to identity. Applied Cognitive Psychology, 13, 513-526.

SumI, S. (1984). Upside-down presentation of the Johansson moving light-spot pattern. Perception, 13, 283-286.

Tadin, D., Lappin, J. S., Blake, R., \& Grossman, E. D. (2002). What constitutes an efficient reference frame for vision? Nature Neuroscience, 5, 1010-1015.

Thornton, I. M., Rensink, R. A., \& Shiffrar, M. (2002). Active versus passive processing of biological motion. Perception, 31, 837-853.

TROJE, N. F. (2002). Decomposing biological motion: A framework for analysis and synthesis of human gait patterns. Journal of Vision, 2, 371-387.

TROJE, N. F. (2003). Reference frames for orientation anisotropies in face recognition and biological-motion perception. Perception, 32, 201-210.

Vaina, L. M., Lemay, M., Bienfang, D. C., Choi, A. Y., \& Nakayama, K. (1990). Intact "biological motion" and "structure from motion" perception in a patient with impaired motion mechanisms: A case study. Visual Neuroscience, 5, 353-369.

WaLK, R. D., \& Homan, C. P. (1984). Emotion and dance in dynamic light displays. Bulletin of the Psychonomic Society, 22, 437-440.

\section{NOTE}

1. Note that temporal perturbations also result in spatial perturbations. See Hill and Pollick (2000) for possible heuristics on separating spatial versus temporal factors.

(Manuscript received September 15, 2003; revision accepted for publication June 14, 2004.) 